\title{
Microemulsion-based novel transdermal delivery system of tetramethylpyrazine: preparation and evaluation in vitro and in vivo
}

This article was published in the following Dove Press journal:

International Journal of Nanomedicine

8 August 201I

Number of times this article has been viewed

Ji-Hui Zhao

$\mathrm{Li} \mathrm{Ji}$

Hui Wang

Zhi-Qiang Chen

Yong-Tai Zhang

Ying Liu

Nian-Ping Feng

School of Pharmacy, Shanghai University of Traditional Chinese

Medicine, Shanghai, People's

Republic of China
Correspondence: Nian-Ping Feng School of Pharmacy, Shanghai University of Traditional Chinese Medicine, Shanghai, 201202,

People's Republic of China

Tel +862151322198

Fax +862151322198

Email npfeng@hotmail.com
Objective: To deliver 2,3,5,6-tetramethylpyrazine (TMP) in a relatively large dose through a transdermal route and facilitate the practical application of microemulison in transdermal drug delivery.

Methods: The pseudo-ternary phase diagram for microemulsion regions was constructed using isopropyl myristate as oil phase, Labrasol ${ }^{\circledR}$ as surfactant, and Plurol ${ }^{\circledR}$ Oleique CC 497 as cosurfactant. A uniform experimental design was applied for formulation optimization. In vitro skin permeation experiments of six formulations were undertaken with TMP transdermal patch (EUDRAGIT ${ }^{\circledR}$ E100 as matrix) and TMP saturated solution as controls. We prepared TMP-oil dispersed in water-ethylene vinyl acetate-transdermal therapeutic system (TMP-O/W-EVA-TTS) with microemulsion as reservoir and EVA membrane as release liner; pharmacokinetic and brain distribution studies in rats were conducted with TMP transdermal patches as control.

Results: The skin fluxes of TMP from microemulsions were 8.2- to 26.7-fold and 0.9- to 4.7-fold higher than those of TMP transdermal patch and TMP saturated solution, respectively, and were strongly affected by the microemulsion composition. The improvement in TMP solubility as well as the skin permeation enhancement effect of microemulsion components contributed mainly to transdermal delivery facilitation. In the pharmacokinetic study, the relative bioavailability of TMP-O/W-EVA-TTS was 350.89\% compared with the TMP transdermal patch. Higher and more stable TMP contents in rat plasma were obtained after administration of TMP-O/WEVA-TTS than after application of TMP transdermal patch. In the brain distribution study, higher rate and extent of TMP distribution to brain, and lower rate of TMP clearance from brain were observed after transdermal administration of TMP-O/W-EVA-TTS than after application of TMP transdermal patch.

Conclusion: The novel transdermal delivery system prepared in this study showed a remarkable skin permeation improvement of microemulsion and facilitated its practical application in transdermal drug delivery. With this system as a vehicle, a relatively large dose of TMP could enable successful drug delivery via the transdermal route.

Keywords: TMP, microemulsion, EVA membrane, transdermal delivery, pharmacokinetics, brain distribution

\section{Introduction}

2,3,5,6-tetramethylpyrazine (TMP), an active ingredient isolated in 1957 from the traditional medicinal herb Ligusticum wallichii Franch, has been widely used in China to treat cardiovascular and cerebrovascular diseases. ${ }^{1,2}$ In the past 2 decades, TMP has been shown to have a beneficial effect on ischemic brain injury in animal experiments and in clinical practice. ${ }^{3-5}$ The mechanism of TMP neuroprotective effects might be related to antioxidation, reduction of calcium overload, and inhibition of the inflammatory response. ${ }^{6}$ 
The therapeutic time window of TMP reducing cerebral ischemia/reperfusion injury in rats was reported to be within a 4-hour time period post-transient focal stroke. ${ }^{7}$ It was reported recently that following intravenous injection to rats, TMP penetrated through the blood-brain barrier within 10 minutes but was eliminated rapidly. Two hours after administration, plasma level of TMP was undetectable. ${ }^{8,9}$ Similarly, rapid distribution into, and elimination from, brain tissue of TMP were reported after intranasal administration to rats. ${ }^{10,11}$ Oral administration of TMP is known to exhibit extensive firstpass metabolism, low oral bioavailability ( $10 \%$ to $30 \%$ ), and short biological half-life of $0.5-2$ hours, suggesting a possible rapid elimination of TMP from brain. ${ }^{12,13}$ To maintain therapeutic blood levels and subsequent therapeutic levels in brain tissue, TMP hydrochloride injection $(40 \mathrm{mg} / 2 \mathrm{~mL})$ or TMP phosphate injection $(50 \mathrm{mg} / 2 \mathrm{~mL})$ is diluted with 250 to $500 \mathrm{~mL}$ of isotonic saline or glucose solution and intravenously infused for 4-6 hours. ${ }^{14}$ For maintenance therapy, controlled release of TMP through the transdermal route might be meaningful for improving effectiveness as well as compliance.

Recently, reservoir type and ethosome-based patch for transdermal delivery of TMP were reported, respectively. ${ }^{15,16}$ However, how to enhance the skin permeation for delivering TMP to the systemic circulation in therapeutic relevant levels is still a critical issue in the development of transdermal formulations, considering the formidable barrier effect of the stratum corneum on foreign substances and the relatively large dose of TMP required.

Microemulsion is defined as a dispersion consisting of oil, surfactant, cosurfactant, and aqueous phase, which is a single optically isotropic and thermodynamically stable liquid solution with a droplet diameter usually within the range of $10-100 \mathrm{~nm} .{ }^{17}$ Microemulsion enhances drug solubility and thermodynamic activity toward the skin, enhances permeation activity of its components, is easy to formulate, and fosters the system's thermodynamic stability. ${ }^{18,19}$ During recent decades numerous studies have suggested that microemulsion has the potential to increase cutaneous delivery of both hydrophilic and lipophilic drugs compared with conventional vehicles. However, the practical application of microemulsions as transdermal delivery vehicles was limited by their good fluidity. Thus, most of the studies were restricted to in vitro skin permeation experiments, and few in vivo investigations were conducted. ${ }^{20}$

To facilitate the transdermal delivery of relatively large doses of active pharmaceutical ingredients and promote the practical application of microemulsions as transdermal delivery vehicles, a novel TMP transdermal therapeutic system (TTS) with oil in water $(\mathrm{O} / \mathrm{W})$ microemulsion as reservoir and ethylene vinyl acetate (EVA) membrane as release liner (TMP-O/W-EVA-TTS) was prepared and evaluated mainly through in vitro skin permeation experiments and pharmacokinetic and brain distribution studies in rats with TMP transdermal patch (EUDRAGIT ${ }^{\circledR}$ E100 as matrix) as control.

\section{Materials and methods Materials}

TMP was obtained from Nanjing Zelang Medical Technology Co, Ltd, Nanjing, China. Caprylocaproyl macrogol-8 glycerides (Labrasol ${ }^{\circledR}$ ) and 1-polyglyceryl-3 dioleate (Plurol ${ }^{\circledR}$ Oleique CC 497) were donated from Gattefossè, Lyon, France. Isopropyl myristate (IPM) was purchased from Shanghai Chemical Reagent Corporation, Shanghai, China. EVA membrane with 19\% VA content (3M Cotran ${ }^{\text {TM }}$ 9728) was obtained from 3M Pharmaceuticals, St Paul, MN. Xanthan gum, pharmaceutical grade, was purchased from Shandong Fufeng Fermentation Co, Ltd, Shandong, China. Polymethacrylates (EUDRAGIT ${ }^{\circledR}$ E100) was donated from BASF, Ludwigshafen, Germany. Other chemicals are HPLC or analytical grade.

\section{Construction of pseudo-ternary phase diagram}

The water titration method was applied for the phase diagram construction. To a certain amount of IPM and mixture of Labrasol ${ }^{\circledR}$ (surfactant, S) with Plurol ${ }^{\circledR}$ Oleique CC 497 (cosurfactant, CoS), distilled water was added dropwise at $25^{\circ} \mathrm{C}$ under mild magnetic stirring. The weight ratio of $\mathrm{S}$ to $\mathrm{CoS}(\mathrm{Km}=3)$ was fixed at 3:1. The weight ratios of IMP to mixture of $\mathrm{S}$ with $\mathrm{CoS}$ ranged from 9.5:0.5, 9:1, 8.5:1.5, 8:2, 7.5:2.5, 7:3, 6:4, 5:5, 4:6, $3: 7,2: 8$, to $1: 9$. The system with low viscosity and clear appearance was considered to be microemulsion. The critical points between microemulsion region and other phase regions were determined when the appearance of the system changed from clear to turbid, and vice versa. The microemulsion regions were defined as $\mathrm{O} / \mathrm{W}$ or $\mathrm{O} / \mathrm{W}$ type according to oil and water weight ratios. The obtained phase diagram is shown in Figure 1.

\section{Formulation optimization}

To optimize microemulsion formulation, a 2-factor, 6-level uniform experimental design was employed. 


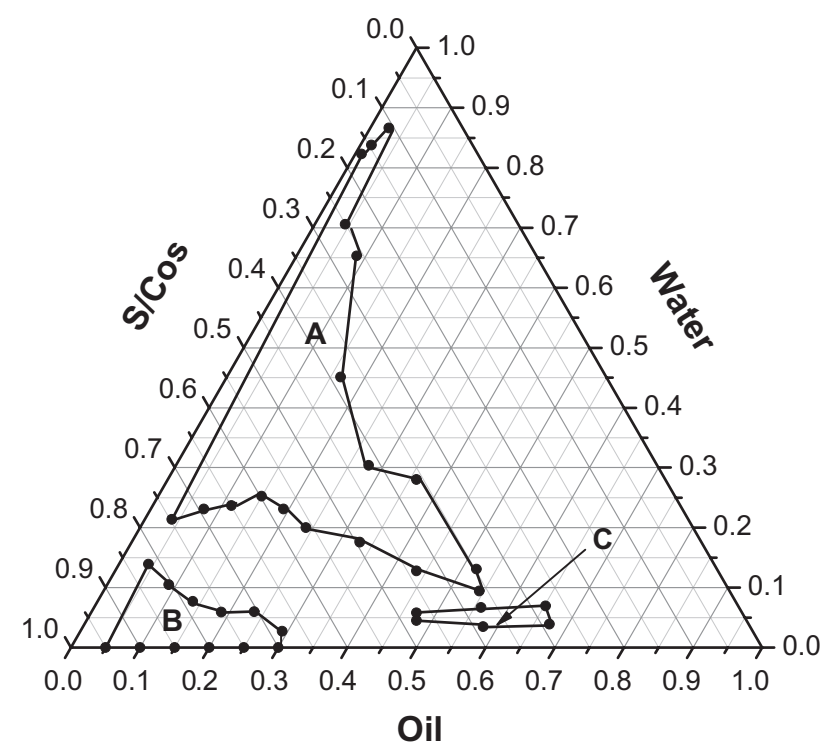

Figure I Pseudo-ternary phase diagram of microemulsion composed of isopropyl myristate, surfactant $\left(\right.$ Labraso $\left.^{\circledR}\right)$ and CoSurfactant (Plurol ${ }^{\circledR}$ Oleique CC 497), $\mathrm{Km}=3: 1$, and water. (A) O/W microemulsion zone; (B) and (C) W/O microemulsion zones. Abbreviations: $\mathrm{O} / \mathrm{W}$, oil in water; W/O, water in oil.

The content of oil $\left(\mathrm{X}_{1}\right)$, weight percentage of surfactant and cosurfactant mixture, $\mathrm{Km}=3,\left(\mathrm{X}_{2}\right)$, and TMP skin flux in vitro $\left(\mathrm{Y}_{1}\right)$ were selected as factors and response, respectively.

Since $\mathrm{O} / \mathrm{W}$ microemulsions were reported to provide significantly greater fluxes for both hydrophobic and hydrophilic drugs, six formulations were selected in this region, and six runs were conducted. ${ }^{21}$ The experimental design and the compositions of the tested formulations are presented in Tables 1 and 2, respectively.

The data from the skin permeation experiments in vitro were fitted to a second-order polynomial model. Data fitting was also undertaken with equilibrium solubility of TMP in microemulsions $\left(\mathrm{Y}_{2}\right)$ as response, since solubility improvement was reported to possibly play a

Table I Uniform experimental design for microemulsion formulation optimization

\begin{tabular}{|c|c|c|}
\hline \multirow[t]{2}{*}{ Experiment } & \multicolumn{2}{|l|}{ Factors } \\
\hline & $\begin{array}{l}X_{1} \text { (weight ratio } \\
\text { of oil, \%) }\end{array}$ & $\begin{array}{l}X_{2} \text { (weight ratio } \\
\text { of the mixture of S } \\
\text { and } \operatorname{CoS}, \mathrm{Km}=3, \% \text { ) }\end{array}$ \\
\hline $\bar{I}$ & 6.0 & 38.0 \\
\hline 2 & 7.0 & 56.0 \\
\hline 3 & 8.0 & 32.0 \\
\hline 4 & 9.0 & 50.0 \\
\hline 5 & 10.0 & 30.0 \\
\hline 6 & 11.0 & 44.0 \\
\hline
\end{tabular}

major role in enhancing drug transdermal delivery by microemulsions..$^{22,23}$

Formulation 6 with TMP loading of $0.6 \%, 1.0 \%$, and $2.0 \%(\mathrm{~W} / \mathrm{W})$ was prepared. The influence of TMP loading on skin flux in vitro was evaluated.

\section{Characterization of the prepared microemulsions}

The viscosity of the prepared microemulsions was measured using a Brookfield DV-II + digital viscometer (Brookfield Engineering Laboratories Inc, MA, USA) with a No. 1 rotor set at $100 \mathrm{rpm}$, and the average droplet sizes were measured with photo-correlation spectroscopy through a Nano ZS90 Zetasizer (Malvern Instruments Ltd, Worcestershire, UK). The morphology of TMP-loaded microemulsion (formulation 6) was observed with transmission electron microscopy (TEM) (JEM-1230; JEOL, Tokyo, Japan). Samples were placed on a film-coated copper grid and allowed to stand for 2 minutes and excess fluid was removed with filter paper.

The equilibrium solubility of TMP in formulations 1 to 6 was determined. First, blank microemulsions were prepared by adding distilled water dropwise to the mixture of IPM, Labrasol ${ }^{\circledR}$, and Plurol ${ }^{\circledR}$ Oleique CC 497 under mild magnetic stirring at room temperature. Then, excess TMP was added to each blank microemulsion. The obtained TMP-loaded microemulsions were sealed and kept under mild magnetic stirring at room temperature for 72 hours. After centrifugation at 10,000 rpm for 10 minutes, TMP concentrations in the supernatants were measured by HPLC after suitable dilution with methanol.

\section{Skin permeation experiments in vitro}

Franz type diffusion cells were applied for the skin penetration experiments. The effective penetration area was $1.7 \mathrm{~cm}^{2}$ and the receptor compartment volume was $16 \mathrm{~cm}^{3}$. Fresh excised abdominal skins of male Sprague-Dawley rats were used as a barrier membrane. To the donor compartments, $3 \mathrm{~mL}$ TMP saturated solution or TMP-loaded microemulsion was added, and the compartments were sealed with parafilm. TMP transdermal patches with TMP loading of $10 \mathrm{mg}$ and effective penetration area of $6 \mathrm{~cm}^{2}$ were prepared and used as control. The TMP transdermal patches were attached to the skins, trimmed, and fixed together with donor compartments. To ensure sink conditions, $10 \%$ ethanol in normal saline $(\mathrm{v} / \mathrm{v})$ was used as receptor medium. 
Table 2 Compositions and drug loadings of formulations I to 6 of microemulsions

\begin{tabular}{|c|c|c|c|c|c|c|}
\hline \multirow[t]{2}{*}{ Formulation } & \multicolumn{4}{|c|}{ Weight fractions of components $(\%, w / w)$} & \multirow{2}{*}{$\begin{array}{l}\text { TMP loading } \\
(\%, w / w)\end{array}$} & \multirow{2}{*}{$\begin{array}{l}\text { Azone } \\
(\%, w / w)\end{array}$} \\
\hline & Labrasol $^{\circledR}$ & Plurol $^{\circledR}$ Oleique CC & IPM & Water & & \\
\hline I & 28.5 & 9.5 & 6.0 & 56.0 & & \\
\hline 2 & 42.0 & 14.0 & 7.0 & 37.0 & & \\
\hline 3 & 24.0 & 8.0 & 8.0 & 60.0 & 2.0 & 1.0 \\
\hline 4 & 37.5 & 12.5 & 9.0 & 41.0 & & \\
\hline 5 & 22.5 & 7.5 & 10.0 & 60.0 & & \\
\hline 6 & 33.0 & 11.0 & 11.0 & 45.0 & & \\
\hline
\end{tabular}

Abbreviations: IPM, isopropyl myristate; TMP, 2,3,5,6-tetramethylpyrazine.

Samples of $1.0 \mathrm{~mL}$ were taken from receptor compartments at predetermined time intervals, and replenished with blank medium of the same volume at $32^{\circ} \mathrm{C}$. TMP concentrations in obtained samples were determined by HPLC.

The cumulative amounts of TMP permeated through excised rat skin were calculated by the equation:

$$
\mathrm{Qs}=\frac{\mathrm{V}}{\mathrm{S}} \times \mathrm{C}_{n}+\sum_{\mathrm{n}-1}^{\mathrm{n}-1} \frac{\mathrm{V}_{i}}{\mathrm{~S}} \mathrm{C}_{i}
$$

where $\mathrm{C}_{n}$ is the TMP concentration in the receptor medium at each sampling time, $\mathrm{C}_{i}$ is the drug concentration of the sample, and $\mathrm{V}$ and $\mathrm{V}_{i}$ are the volumes of the receptor medium and the sample, respectively. $\mathrm{S}$ is the effective diffusion area $\left(\mathrm{S}=1.7 \mathrm{~cm}^{2}\right)$. The cumulative amount of TMP was plotted as a function of time. The permeation rate of TMP as steady state $\left(J_{S}, \mu \mathrm{g} / \mathrm{cm}^{2} / \mathrm{h}\right)$ through rat skin was calculated from the slope of the linear portion of the cumulative amount per unit area versus time plot.

\section{Drug-release experiments in vitro}

A release experiment in vitro of TMP from formulation 6 at TMP loading of $1.0 \%(\mathrm{~W} / \mathrm{W})$ was undertaken. The experimental conditions were the same as those for the skin permeation experiments in vitro, except for the replacement of rat skins by the EVA membranes as permeation barriers.

\section{Fabrication of TMP-O/W-EVA-TTS}

TMP-O/W-EVA-TTSs were fabricated with microemulsion as reservoir and EVA membrane as release liner. Microemulsion was prepared according to formulation 6, with TMP loading being $1.0 \%(\mathrm{w} / \mathrm{w})$. To facilitate the preparation of TMP-O/WEVA-TTSs, $0.5 \%(\mathrm{w} / \mathrm{w})$ of xanthan gum was added to the microemulsion. The TMP loading and the effective skin permeation area of TMP-O/W-EVA-TTSs were $10 \mathrm{mg}$ and $6 \mathrm{~cm}^{2}$, respectively.

TMP transdermal patches with the same drug loading and effective permeation area as those of TMP-O/W-EVA-TTSs were prepared and employed as control in the pharmacokinetic and brain distribution studies in rats.

\section{Pharmacokinetic study}

Male Sprague-Dawley rats (220-240 g), provided by Shanghai University of TCM Animal Center (Shanghai, China), were randomly divided into TMP-O/W-EVA-TTS and TMP transdermal patch groups $(\mathrm{n}=6)$. TMP-O/W-EVA-TTS was administered transdermally as a single dose to the abdominal area of each rat in the TMP-O/W-EVA-TTS group after carefully shaving the hair. Similarly, TMP transdermal patch was administered to each rat in the TMP-O/W-EVA-TTS group.

After the administration, $300-\mu \mathrm{L}$ blood samples were withdrawn from eye venous plexus and collected into heparinized tubes at $0,1,2,3,4,8,12$, and 24 hours from rats in each group. Plasma samples were separated by immediate centrifugation at $6000 \mathrm{rpm}$ for 5 minutes, and stored at $-70^{\circ} \mathrm{C}$ until analysis.

To a volume of $150 \mu \mathrm{L}$ plasma sample, $15 \mu \mathrm{L}$ of doxofylline as internal standard, $\mathrm{NaOH}$ solution $(1 \mathrm{~N})$, and methanol were added consecutively. The obtained mixture was extracted twice with N-hexane and chloroform $(2: 1, \mathrm{v} / \mathrm{v})$. At each extraction, a total of $450 \mu \mathrm{L} \mathrm{N}$-hexane and chloroform was added and mixed with a vortex mixer for 3 minutes. After centrifugation at $15,000 \mathrm{rpm}$ at $4^{\circ} \mathrm{C}$ for 10 minutes, the supernatants from each plasma sample were combined and $15 \mu \mathrm{L}$ of $\mathrm{HCl}$ in methanol solution (1 N) was added. The solvent was evaporated to dryness under gentle nitrogen stream. Each residue was reconstituted with $50 \mu \mathrm{L}$ of methanol before the determination of TMP content by HPLC.

The measurements of TMP concentrations in rat plasma were carried out with a SHIMADAZU HPLC system equipped with a UV detector, an autosampler, and a reverse phase Dikma Platisil $\mathrm{C}_{18}$ column. The mobile phase was composed of methanol and ammonium chloride buffer (45:55, $\mathrm{v} / \mathrm{v}$ ), and the $\mathrm{pH}$ value of the mobile phase was adjusted to 10.0 . The flow rate was $1.0 \mathrm{~mL} /$ minute. The detection 
Table 3 Characteristics of formulation I to formulation 6 and skin permeation parameters of TMP

\begin{tabular}{|c|c|c|c|c|c|}
\hline \multirow[t]{2}{*}{ Formulation } & \multicolumn{4}{|c|}{ Characteristics of microemulsions } & \multirow{2}{*}{$\begin{array}{l}\text { Skin permeation } \\
\text { parameter } \\
\text { Flux } \\
\left(\mathrm{mg} / \mathrm{cm}^{2} / \mathrm{h}\right)\end{array}$} \\
\hline & $\begin{array}{l}\text { Mean droplet } \\
\text { size }(\mathrm{nm})\end{array}$ & PDI & $\begin{array}{l}\text { Viscosity } \\
\text { (mPa.S) }\end{array}$ & $\begin{array}{l}\text { TMP solubility } \\
(\mathrm{mg} / \mathrm{mL})\end{array}$ & \\
\hline $\mathrm{I}$ & $51.47 \pm 0.25$ & $0.352 \pm 0.015$ & $34.0 \pm 0.4$ & 4.13 & $0.164 \pm 0.022$ \\
\hline 2 & $95.04 \pm 1.30$ & $0.251 \pm 0.015$ & $53.2 \pm 0.5$ & 7.07 & $0.139 \pm 0.004$ \\
\hline 3 & $45.02 \pm 0.37$ & $0.329 \pm 0.003$ & $30.2 \pm 1.1$ & 6.07 & $0.087 \pm 0.012$ \\
\hline 4 & $78.21 \pm 1.20$ & $0.274 \pm 0.004$ & $45.0 \pm 0.6$ & 4.93 & $0.183 \pm 0.019$ \\
\hline 5 & $42.74 \pm 0.13$ & $0.375 \pm 0.011$ & $30.2 \pm 0.5$ & 5.93 & $0.191 \pm 0.023$ \\
\hline 6 & $66.64 \pm 0.18$ & $0.397 \pm 0.002$ & $33.3 \pm 1.6$ & 9.88 & $0.263 \pm 0.027$ \\
\hline TMP transdermal patch & - & - & - & - & $0.009 \pm 0.0007$ \\
\hline TMP saturated solution & - & - & - & 1.90 & $0.046 \pm 0.005$ \\
\hline
\end{tabular}

Abbreviations: PDI, polydispersity index; TMP, 2,3,5,6-tetramethylpyrazine.

wavelength was set at $280 \mathrm{~nm}$, the column temperature was set at $35^{\circ} \mathrm{C}$, and the injection volume was $20 \mu \mathrm{L}$.

\section{Brain distribution study}

The animal grouping and administration were the same as those of pharmacokinetic study, except that 21 instead of six rats were used in each group. At $0,2,4,8,12,16$, and 24 hours after the administration, rats $(\mathrm{n}=3$ for each time point) in each group were sacrificed, and a brain sample from each rat was isolated, rinsed thoroughly with normal saline, and weighed after drying with filter paper. To each tube containing 1 brain sample, normal saline was added at a weight ratio of 2:1. After homogenation for 5 minutes with a hand-held tissue homogenizer in an ice water bath and centrifugation at $10,000 \mathrm{rpm}$ for 5 minutes, brain homogenate samples were obtained and stored at $-70^{\circ} \mathrm{C}$ until analysis.

Brain homogenate samples were prepared by the same procedure as plasma samples except that $100 \mu \mathrm{L}$ of doxofylline, $\mathrm{NaOH}$ solution $(1 \mathrm{~N})$, and methanol were added to $1.0 \mathrm{~mL}$ brain homogenate sample, and a total of $3.0 \mathrm{~mL}$ $\mathrm{N}$-hexane and chloroform was added at each time of extraction, and $100 \mu \mathrm{L}$ of $\mathrm{HCl}$ in methanol solution $(1 \mathrm{~N})$ was added to the supernatant from each brain homogenate sample. The HPLC conditions for TMP contents measurement in rat brain homogenates were the same as those for TMP concentration determination in rat plasma.

\section{Data analysis}

Non-compartment pharmacokinetic analysis of TMP plasma concentrations versus time data were conducted using BAPP2.0 (Bioavailability program package 2.0 , Center for Metabolism and Pharmacokinetics, China Pharmaceutical University, Nanjing, China).

\section{Results and discussion Characterization of the prepared microemulsions}

The mean droplet sizes of the prepared microemulsions ranged from 42.74 to $95.04 \mathrm{~nm}$, and polydispersity index ranged from 0.251 to 0.397 , suggesting small nanosized droplets with relative uniform droplet sizes were obtained following formulations 1 to 6 (Table 3). The microemulsion droplets obtained were spherical and relatively even in size (Figure 2). A trend of viscosity increase was observed when the contents of Labrasol ${ }^{\circledR}$ (surfactant) in the formulations were $>37.5 \%$.

\section{Formulation optimization}

The skin permeation profiles of formulations 1 to 6 of microemulsions compared with TMP transdermal patch and TMP saturated solution are shown in Figure 3, and the skin permeation parameters of TMP are presented in Table 3. An obvious enhancement in skin permeability of TMP was observed by the application of all microemulsions compared with the TMP transdermal patch. The highest skin flux

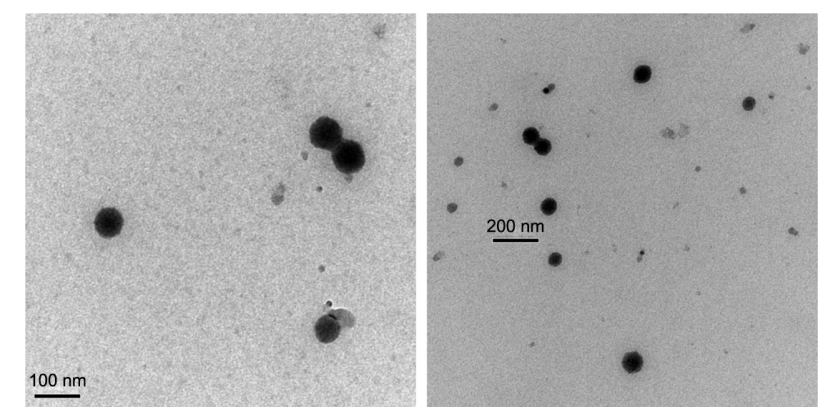

Figure 2 Transmission electron microscopy of tetramethylpyrazine-loaded microemulsion (formulation 6). 


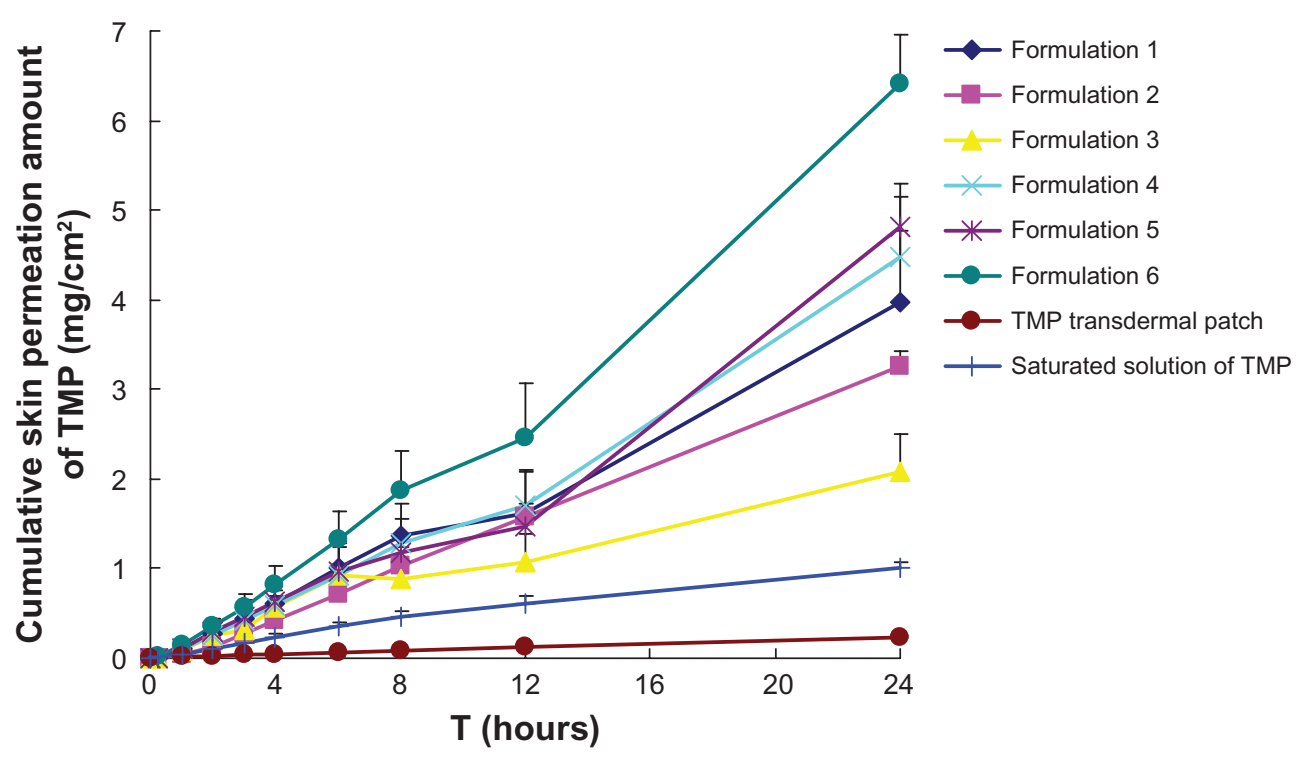

Figure 3 In vitro skin permeation profiles of TMP from formulations I to 6 of microemulsions, TMP loading was 2\% (w/w), with TMP transdermal patch and TMP saturated solution as controls. The weight fractions of components in formulations I to 6 were 6.0, 7.0, 8.0, 9.0, I0.0 and II.0 for IPM; 28.5, 42.0, 24.0, 37.5, 22.5, and 33.0 for Labrasol ${ }^{\circledR}$; and 9.5, 14.0, 8.0, 12.5, 7.5, and II .0 for Plurol ${ }^{\circledR}$ Oleique CC 497, respectively.

Abbreviations: IPM, isopropyl myristate; TMP, 2,3,5,6-tetramethylpyrazine.

of TMP $\left(0.263 \pm 0.027 \mathrm{mg} / \mathrm{cm}^{2} / \mathrm{h}\right)$ was obtained following the application of formulation 6 . The skin fluxes of TMP from microemulsions were 8.2 - to 26.7 -fold higher than from TMP transdermal patch. This result might be due to the much higher diffusion rates of TMP from microemulsions as liquid medium than that from TMP transdermal patch with pressure-sensitive adhesive as solid matrix. Similarly, the skin fluxes of TMP from microemulsions were 0.9- to 4.7-fold higher than those from TMP saturated solution.
The permeation profiles of microemulsions followed zero order release kinetics. The possible explanation is that the surfactant and cosurfactant may exist in each phase of the microemulsion, so that active ingredient can be partly solubilized in external phase, and the depletion of active ingredient in external phase because of permeation into the skin can be supplemented by the release of active ingredient from internal phase. Then the zero order release kinetics and sustained, controlled, prolonged delivery of active ingredient were obtained. ${ }^{24}$

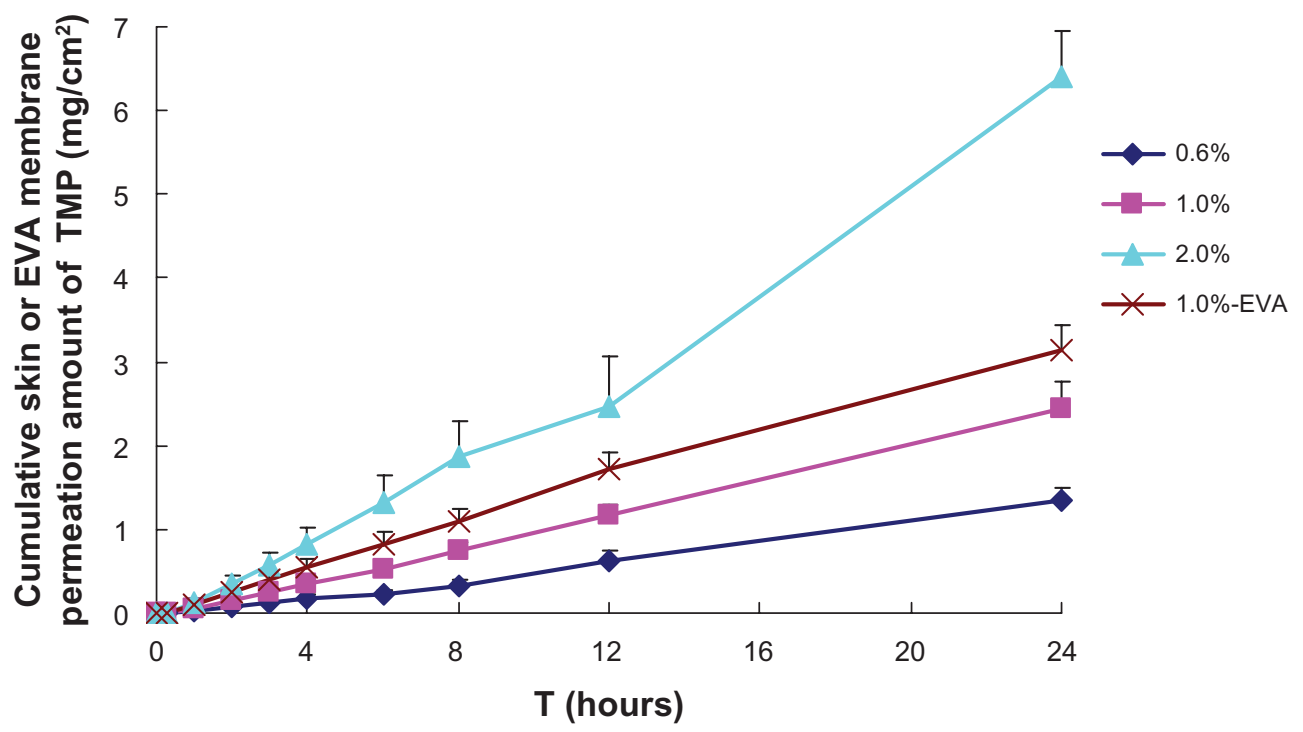

Figure 4 In vitro skin or EVA membrane permeation profiles of TMP from formulation 6 of microemulsions I.0\%-EVA: EVA membrane permeation profile of TMP from formulation 6 , with TMP loading being $1.0 \%(\mathrm{w} / \mathrm{w}) ; 0.6 \%, 1.0 \%$, and $2.0 \%$ : rat skin permeation profiles of TMP from formulation 6 , with TMP loadings being $0.6 \%$, I.0\%, and $2.0 \%(w / w)$, respectively.

Abbreviations: EVA, ethylene vinyl acetate; IPM, isopropyl myristate; TMP, 2,3,5,6-tetramethylpyrazine. 
Table 4 Skin permeation parameters in vitro and characteristics of formulation 6 with different drug loadings

\begin{tabular}{llll}
\hline Drug loading $(\%, w / w)$ & $\begin{array}{l}\text { Saturated amount of TMP } \\
\text { in the donor compartment } \mathbf{( m g )}\end{array}$ & $\begin{array}{l}\text { Residue amount of TMP in the } \\
\text { donor compartment after } \mathbf{2 4} \mathbf{h}(\mathbf{m g})\end{array}$ & $\begin{array}{l}\text { Skin flux of TMP } \\
\left(\mathbf{m g} / \mathbf{c m}^{2} / \mathbf{h}\right)\end{array}$ \\
\hline 0.6 & 29.64 & $15.72 \pm 1.24$ & $0.056 \pm 0.007$ \\
1.0 & 29.64 & $25.86 \pm 3.58$ & $0.103 \pm 0.014$ \\
2.0 & 29.64 & $49.10 \pm 4.96$ & $0.263 \pm 0.027$ \\
\hline
\end{tabular}

Abbreviation: TMP, 2,3,5,6-tetramethylpyrazine.

The second-order polynomial equations with TMP skin flux in vitro $\left(\mathrm{Y}_{1}\right)$, and equilibrium solubility of TMP in microemulsions $\left(\mathrm{Y}_{2}\right)$, respectively, content of IPM $\left(\mathrm{X}_{1}\right)$, and weight percentage of surfactant and cosurfactant mixture ( $\mathrm{S} / \mathrm{Cos}), \mathrm{Km}=3,\left(\mathrm{X}_{2}\right)$ as factors were as follows:

$$
\begin{aligned}
\mathrm{Y}_{1}= & 0.866-20.295 \mathrm{X}_{1}+128.707 \mathrm{X}_{1}^{2} \\
& +0.025 \mathrm{X}_{2}^{2}+1.663 \mathrm{X}_{1} \mathrm{X}_{2}(R=0.952) \\
\mathrm{Y}_{2}= & 16.199-362.690 \mathrm{X}_{1}+2977.834 \mathrm{X}_{1}^{2} \\
& +23.617 \mathrm{X}_{2}^{2}-162.797 \mathrm{X} 1 \mathrm{X} 2(R=0.808)
\end{aligned}
$$

The second-order polynomial equations show that $Y_{1}$ was better correlated than $Y_{2}$ with $X_{1}$ and $X_{2}$. This might be due to the skin permeation enhancement effect of microemulsion components. IPM was reported to be an effective penetration enhancer, and the surfactant and cosurfactant in the microemulsions might reduce the diffusional barrier of the stratum corneum by acting as permeation enhancers. ${ }^{25,26}$

To find out the influence of microemulsion composition on the skin permeation behavior of TMP, grid searches over the whole experimental region were conducted under the guidance of visual basic programs on the basis of secondorder polynomial equations mentioned above. According to the grid searches, an increase of skin flux and TMP solubility in microemulsions were observed with the increase of IPM content as well as the weight fraction of S/CoS. The changing IPM content showed greater influence on TMP skin flux, which was no more than $0.18 \mu \mathrm{g} / \mathrm{cm}^{2} / \mathrm{h}$, when IMP content was $<9 \%(w / w)$. Thus, the improvement in TMP solubility as well as the skin permeation enhancement effect of microemulsion components contributed mainly to transdermal delivery facilitation. This result was in agreement with previous work. ${ }^{27}$

According to earlier reports, drug flux decrease was observed with the increase in amount of surfactant in microemulsions, ${ }^{28,29}$ possibly because of a decreased thermodynamic activity of the drug in microemulsion at higher surfactant concentrations. Another possibility was that drug diffusion through the double layer microemulsion might be a rate-determining step, as viscosity plays an important role in controlling the drug release into the receptor..$^{30}$ However, in this study, the skin flux increase was observed with the rise in the weight fraction of $\mathrm{S} / \mathrm{CoS}$. The first mechanism might be because the viscosity of the systems prepared in this study did not increase as much with increasing amount of surfactant as that reported previously. The second possibility was that medium-weight fractions of surfactant ranging from $22.5 \%$ to $42.0 \%$ were chosen for this study. In addition, the mixture of

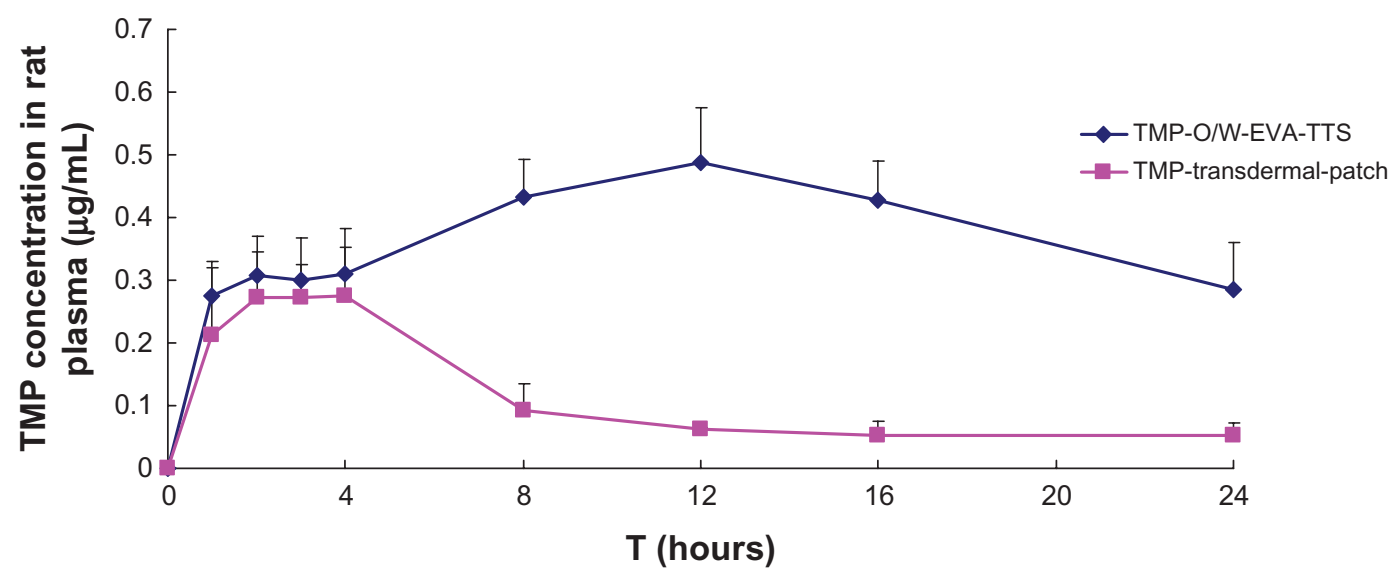

Figure 5 Mean concentrations of TMP in rat plasma versus time profiles from TMP-O/W-EVA-TTS and TMP transdermal patch.

Abbreviations: TMP, 2,3,5,6-tetramethylpyrazine; TMP-O/W-EVA-TTS, tetramethylpyrazine-oil dispersed in water-ethylene vinyl acetate-transdermal therapeutic system. 
Table 5 Pharmacokinetic parameters of TMP after a single-dose transdermal administration of TMP-O/W-EVA-TTS $(10 \mathrm{mg}$ of TMP in $\left.6 \mathrm{~cm}^{2}\right)$ to each rat in group $A(n=6)$ and TMP transdermal patch $\left(10 \mathrm{mg}\right.$ of TMP in $\left.6 \mathrm{~cm}^{2}\right)$ to each rat in group $B(n=6)$

\begin{tabular}{llc}
\hline Parameters & TMP-O/W-EVA-TTS & $\begin{array}{l}\text { TMP transdermal } \\
\text { patch }\end{array}$ \\
\hline $\mathrm{C}_{\max }(\mu \mathrm{g} / \mathrm{mL})$ & $0.49 \pm 0.09$ & $0.29 \pm 0.07$ \\
$\mathrm{~T}_{\max }(\mathrm{h})$ & 12.00 & $2.67 \pm 0.58$ \\
$\mathrm{AUC}\left(\mathrm{h}^{*} \mu \mathrm{g} / \mathrm{mL}\right)$ & $9.0495 \pm 1.2368^{* *}$ & $2.579 \pm 0.723$ \\
$\mathrm{MRT}(\mathrm{h})$ & $12.1939 \pm 0.37 \mathrm{I} 2^{* *}$ & $7.886 \pm 0.35 \mathrm{I}$ \\
$\mathrm{Cl}(\mathrm{L} / \mathrm{h})$ & $0.676 \pm 0.207^{*}$ & $3.324 \pm 0.997$ \\
Relative & 350.89 & \\
bioavailability (\%) & & \\
\hline
\end{tabular}

Notes: $*$ and $* *$ represent $P<0.05$ and $P<0.01$, respectively, compared with TMP transdermal patch.

Abbreviations: $\mathrm{AUC}$, area under the curve; $\mathrm{Cl}$, clearance; $\mathrm{MRT}$, mean residence time; TMP, 2,3,5,6-tetramethylpyrazine; TMP-O/W-EVA-TTS, tetramethylpyrazineoil dispersed in water-ethylene vinyl acetate-transdermal therapeutic system.

surfactant and cosurfactant, but not surfactant alone, served as a factor in the experiments of uniform design conducted in this study.

The permeation rate of TMP was almost proportional to drug loading (Figure 4). To give a possible explanation of this phenomenon, the saturated amount of TMP in the donor compartment and the residue amount of TMP after 24 hours were calculated (Table 4). The reduction of TMP skin flux with decrease in TMP loading might be due to the depletion of TMP in the donor compartment and subsequent decline of driving concentration. However, in consideration of the physical instability when the drug loading was $2.0 \%$, namely crystallization of TMP during storage, the final loading of TMP in formulation 6 was $1.0 \%$.

A higher release flux of TMP than skin permeation flux was observed (Figure 4), indicating that the rate-limiting step in TMP skin permeation process was its permeation through the skin rather than its release from the vehicle. This result is in agreement with a previous report. ${ }^{31}$

\section{Pharmacokinetic and brain distribution studies}

Mean rat plasma concentrations versus time profiles and the pharmacokinetic parameters of TMP are shown in Figure 5 and Table 5, respectively. Within 4 hours after the administration, TMP-O/W-EVA-TTS and TMP transdermal patch showed comparable plasma concentrations. However, after that, TMP-O/W-EVA-TTS showed an obvious increase of TMP contents in plasma, $\mathrm{C}_{\text {max }}$ of TMP was reached in 12 hours, and high levels of TMP were maintained in 24 hours. In contrast, TMP transdermal patch showed considerable reduction of TMP contents in plasma, and low levels of TMP were observed from 8 to 24 hours after the administration. The overall mean value of $\mathrm{AUC}_{0-\mathrm{t}}$ of TMP-O/W-EVA-TTS was 2.5 times higher than that of TMP-O/W-EVA-TTS $(P<0.01)$. The subsequent relative bioavailability of TMP-O/W-EVA-TTS was $350.89 \%$. Similarly, significantly prolonged $(P<0.01)$ MRT and reduced $(P<0.05) \mathrm{Cl}$ of TMP-O/W-EVA-TTS were observed.

TMP-O/W-EVA-TTS showed higher TMP contents than TMP transdermal patch during the whole experimental period, namely within 24 hours after administration (Figure 6). This result suggests the higher distribution rate and extent of TMP to brain, and lower clearance of TMP from brain following the transdermal administration of TMP-O/W-EVA-TTS, than from administration of TMP transdermal patch.

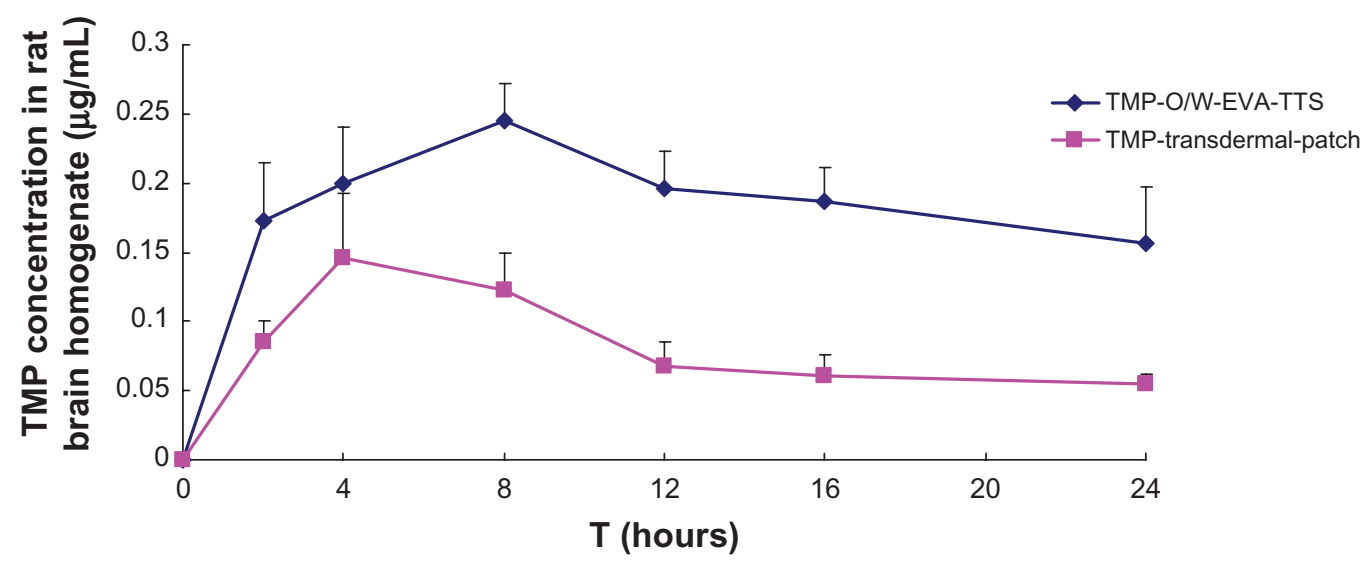

Figure 6 Mean concentrations of TMP in rat brain homogenate versus time profiles from TMP-O/W-EVA-TTS and TMP transdermal patch. Abbreviation: TMP, 2,3,5,6-tetramethylpyrazine; TMP-O/W-EVA-TTS, tetramethylpyrazine-oil dispersed in water-ethylene vinyl acetate-transdermal therapeutic system. 


\section{Conclusion}

The novel transdermal delivery system prepared in this study showed a remarkably improved skin permeation of microemulsion and facilitated its practical application in transdermal drug delivery. With this system as a vehicle, a relatively large dose of TMP could enable successful drug delivery via the transdermal route.

\section{Acknowledgments}

This work was supported by a grant (J50302) from Shanghai Education Committee, Shanghai Natural Science Foundation Project (10ZR1430100) and Shanghai Subject Chief Scientist Program (10XD14303900) from Science and Technology Commission of Shanghai Municipality, and Program (NCET08-0898) for New Century Excellent Talents of the State Education Ministry, P. R. China.

\section{Disclosure}

The authors report no conflicts of interest in this work.

\section{References}

1. Chun-sheng L, Hsiao-meng Y, Yun-hsiang H, et al. Radix salviae miltiorrhizae and Rhizoma ligustici wallichii in coronary heart disease. Chin Med J (Engl). 1978;4(1):43-46.

2. Guo SK, Chen KJ, Qian ZH, et al. Tetramethylpyrazine in the treatment of cardiovascular and cerebrovascular diseases. Planta Med. 1983;47(2):89.

3. Ho WK, Wen HL, Lee CM. Tetramethylpyrazine for treatment of experimentally induced stroke in Mongolian gerbils. Stroke. 1989;20(1):96-99.

4. Liao SL, Kao TK, Chen WY, et al. Tetramethylpyrazine reduces ischemic brain injury in rats. Neurosci Lett. 2004;372(1-2):40-45.

5. Li Qing-an, Fang Ming, Qi Jingjing. Clinical research of protective effect of tetramethylpyrazine on cerebral ischemia/reperfusion injury. Modern Journal of Integrated Traditional Chinese and Western Medicine. 2011;20(10):1177-1178. Chinese.

6. Tsung-Kuei Kao, Yen-Chuan Ou, Jong-song Kuo, et al. Neuroprotection by tetramethylpyrazine against ischemic brain injury in rats. Neurochem Int. 2006;48:166-176.

7. Zhu XL, Xiong LZ, Wang Q, et al. Therapeutic time window and mechanism of tetramethylpyrazine on transient focal cerebral ischemia/ reperfusion injury in rats. Neurosci Lett. 2009;449(1):24-27.

8. Liang CC, Hong CY, et al. Measurement and pharmacokinetic study of tetramethylpyrazine in rat blood and its regional brain tissue by highperformance liquid chromatography. J Chromatogr B Biomed Sci Appl. 1999;724(2):303-309.

9. Tsai TH, Liang C. Pharmacokinetics of tetramethylpyrazine in rat blood and brain using microdialysis. Int J Pharm. 2001;216(1-2):61-66.

10. Mei D, Mao S, Sun W, et al. Effect of chitosan structure properties and molecular weight on the intranasal absorption of tetramethylpyrazine phosphate in rats. Eur J Pharm Biopharm. 2008;70(3):874-881.
11. Feng J, Li F, Zhao Y, et al. Brain pharmacokinetics of tetramethylpyrazine after intranasal and intravenous administration in awake rats. Int $J$ Pharm. 2009;375(1-2):55-60.

12. Cai W, Dong SN, Lou YQ. HPLC determination of tetramethylpyrazine in human serum and its pharmacokinetic parameters. Yaо Хие Хие Bao. 1989;24(12):881-886. Chinese.

13. Lou YQ, Zhang H, Cao X, et al. The pharmacokinetics and disposition of tetramethylpyrazine phosphate in dogs and rats. Yao Xuе Xue Bao. 1986;21(7):481-487. Chinese.

14. Sun BL. Clinical use of tetramethylpyrazine: a review. Chin J Integr Tradit West Med. 1994;14:465-468. Chinese.

15. Qi X, Liu RR, Sun D, et al. Convolution method to predict drug concentration profiles of 2,3,5,6-tetramethylpyrazine following transdermal application. Int J Pharm. 2003;259(1-2):39-45.

16. Liu X, Liu H, Liu J, et al. Preparation of a ligustrazine ethosome patch and its evaluation in vitro and in vivo. Int J Nanomedicine. 2011;6:241-247.

17. Tenjarla S. Microemulsions: an overview and pharmaceutical applications. Crit Rev Ther Drug Carrier Syst. 1999;16:461-521.

18. Podlogar F, Bester Rogac M, Gasperlin M. The effect of internal structure of selected water-Tween 40-Imwitor 308-IPM microemulsions on ketoprofene release. Int J Pharm. 2005;302:68-77.

19. Kogan A, Garti N. Microemulsions as transdermal drug delivery vehicles. Adv Colloid Interface Sci. 2006;123-126:369-385.

20. Kreilgaard M. Influence of microemulsions on cutaneous drug delivery. Adv Drug Deliv Rev. 2002;54(Suppl 1):S77-S98.

21. Lee PJ, Langer R, Shastri VP. Novel microemulsion enhancer formulation for simultaneous transdermal delivery of hydrophilic and hydrophobic drugs. Pharm Res. 2003;20(2):264-269.

22. Zhao X, Liu JP, Zhang X, Li Y. Enhancement of transdermal delivery of theophylline using microemulsion vehicle. Int J Pharm. 2006; 327(1-2):58-64.

23. El Maghraby GM. Transdermal delivery of hydrocortisone from eucalyptus oil microemulsion: effects of CoSurfactants. Int J Pharm. 2008;355(1-2):285-292.

24. Chen H, Chang X, Weng T, et al. A study of microemulsion systems for transdermal delivery of triptolide. J Control Release. 2004;98(3): $427-436$.

25. Melinda Goldberg-Cettina, Puchun Liu, James Nightingale, et al. Enhanced transdermal delivery of estradiol in vitro using binary vehicles of isopropyl myristate and short-chain alkanols. Int J Pharm. 1995; 114(2):237-245

26. Peltola S, Saarinen-Savolainen P, Kiesvaara J, et al. Microemulsions for topical delivery of estradiol. Int J Pharm. 2003;254(2):99-107.

27. Kreilgaard M, Pedersen EJ, Jaroszewski JW. NMR characterization and transdermal drug delivery potential of microemulsion systems. J Control Release. 2000;69(3):421-433.

28. Huang YB, Lin YH, et al. Transdermal delivery of capsaicin derivativesodium nonivamide acetate using microemulsions as vehicles. Int $J$ Pharm. 2008;349(1-2):206-211.

29. Rhee YS, Choi JG, Park ES, et al. Transdermal delivery of ketoprofen using microemulsions. Int J Pharm. 2001;228(1-2):161-170.

30. Ho HO, Hsiao CC, Sheu MT. Preparation of microemulsions using polyglycerol fatty acid esters as surfactant for the delivery of protein drugs. J Pharm Sci. 1996;85(2):138-143.

31. Montenegro L, Carbone C, Puglisi G. Vehicle effects on in vitro release and skin permeation of octylmethoxycinnamate from microemulsions. Int J Pharm. 2011;405(1-2):162-168.
International Journal of Nanomedicine

\section{Publish your work in this journal}

The International Journal of Nanomedicine is an international, peerreviewed journal focusing on the application of nanotechnology in diagnostics, therapeutics, and drug delivery systems throughout the biomedical field. This journal is indexed on PubMed Central, MedLine, CAS, SciSearch $₫$, Current Contents ${ }^{\circledR} /$ Clinical Medicine,

\section{Dovepress}

Journal Citation Reports/Science Edition, EMBase, Scopus and the Elsevier Bibliographic databases. The manuscript management system is completely online and includes a very quick and fair peer-review system, which is all easy to use. Visit http://www.dovepress.com/ testimonials.php to read real quotes from published authors. 